\section{Blast Overpressure Induced Pulmonary and Intestinal Damage is Ameliorated by Post-injury Decay Accelerating Factor Injection}

\begin{abstract}
James E Campbell', Thomas Oh'1, Brady J Hurtgen'1, Debra M Niemeyer $^{1}$, Forest R Sheppard ${ }^{2}$ and Jurandir J Dalle Lucca ${ }^{1 *}$
\end{abstract}

${ }^{1}$ Tri-Services Research Laboratory, 59 ${ }^{\text {th }}$ Medical Wing, Joint Base San Antonio, Fort Sam Houston, San Antonio, Texas, USA

${ }^{2}$ Naval Medical Research Unit, Joint Base San Antonio, Fort Sam Houston, San Antonio, Texas, USA

\begin{abstract}
Blast Overpressure (BOP) inflicts severe immediate physical and continuous inflammatory injury upon air/gas filled-hollow organs, i.e., lungs and intestines. Sprague-Dawley rats were subjected to BOP (120 kilopascals) to study military relevant injuries. Recombinant human DAF (rhDAF) was administered $(50 \mu \mathrm{g} / \mathrm{kg}$ body weight) 30 minutes post-blast to simulate expected emergency care chronology. The rhDAF treatment group was compared to 1) sham control group- no BOP exposure with no saline injection, and 2) BOP with saline injection group (30 minutes post-blast). Within each group, animals underwent recovery (simulated or veritable) and were sacrificed at 3, 24 and 48 hours post-blast. Lung and small intestine tissue samples were prepared for pathology injury assessments, microscopic evaluations and immunohistochemistry assessment of tissue marker deposition (rhDAF, complement C5, Neutrophil Elastase (NE), and caspase-1). BOP resulted in pulmonary and intestinal damage that was mitigated by post-blast administration of rhDAF. Complement activator C5/C5a, NE, and caspase-1 increased during 3 to 48 hours following BOP. rhDAF treatment significantly reduced levels of $\mathrm{C} 5 / \mathrm{C} 5 \mathrm{a}$, and $\mathrm{NE}$ after their induction, but did not alter caspase- 1 levels. These novel findings suggest early complement inhibition via rhDAF administration is a promising new therapeutic strategy to decrease BOP-induced pulmonary and intestinal tissue injury.
\end{abstract}

Keywords: Blast overpressure; Complement activation; Decay accelerating factor; Lung; Rat

*Corresponding author: Jurandir J Dalle Lucca, Joint Inflammation Modulation of Trauma Program (JIMoT), Chief Scientist Office. 59MDW/ST, Wilford Hall Ambulatory Surgical Center, 2200 Berquist Drive, Suite 1 Joint Base San Antonio - Lackland, TX 78236, USA, Tel: +1 2105397399; E-mail: Jurandir.J.DalleLucca.mil@mail.mil

Citation: Campbell JE, Oh T, Hurtgen BJ, Niemeyer DM, Sheppard FR, et al. (2015) Blast Overpressure Induced Pulmonary and Intestinal Damage is Ameliorated by Post-injury Decay Accelerating Factor Injection. J Clin Immunol Immunother 2: 007

Received: May 08, 2015; Accepted: July 03, 2015; Published: July 17, 2015

\section{Introduction}

Pure blast injuries resulting from High-Energy (HE) explosions were first studied during WWI by Hooker. Those studies determined the nature of primary blast injury to be independent of secondary (flying "missiles" of shrapnel and debris), and tertiary (the propelling blast wind and subsequent impact). Outside of a zone of death, described as a short radius of proximity emanating out from the point of explosion, another zone exists that promotes a shock syndrome of blood pressure collapse which indicate some physical injury, however, without any external manifestation [1]. Lung and visceral organ hemorrhage was common in his blast model animals whereas intracranial lesions were absent. WWII studies reported that Blast Overpressure (BOP) waves injure the lungs via impact with the body wall [2]. Case studies at that time supported findings that lungs were the primary organ injured by a BOP yet further suggested ubiquitous damage throughout lung tissue through gross examination assessment of cases that went to necropsy [3]. More recent studies have described these injuries as being owed to the great heterogeneity of solids, liquids, and gasses all occupying a small space in which BOP effects each in unique fashion. However, the primary cause of injury has been shown to be the result of rapid compression and expansion of the gaseous component of anatomical structure [4]. Gastrointestinal tract tissues and organs are also highly susceptible to BOP due to retention and generation of gasses [5,6]. A thorough review of blast-related injury is available [7].

Only from HE explosives does a BOP wave propagate that may induce a primary blast injury. HE explosions occur on American soil in both deliberate and accidental manner, i.e., HE explosions occurred at the 1993 world trade center bombing, the 1998 Alfred P Murray building/Oklahoma city bombing, and constituted the disaster of the 2012 city of West, Texas fertilizer plant. HE explosions are ever increasing in incidence on the global scale with continued blasts in Israeli-Palestinian conflicts and the threat of terrorist attacks in the developed world is constant: London 2005 [8,9], Madrid 2004 [10], and Moscow 2010 [11]. Blast injuries, simulated by the immediately sub-lethal yet tissue-damaging model employed in this manuscript, are a real and present danger with which few civilian physicians have experience in treating [12].

BOP pulmonary and intestinal tissue injury shows general similarity to Ischemia/Reperfusion (I/R) injury in its subsequent increased inflammation and delayed injury response. Complement inhibition therapy have been demonstrated to decrease severity of I/R injuries to these tissues $[13,14]$. Decay Accelerating Factor (DAF) is a naturally present complement-inhibitory protein in humans that binds cells and prevents assembly of both $\mathrm{C} 4 \mathrm{~b} 2 \mathrm{a}$ and $\mathrm{C} 3 \mathrm{bBb}$, the classical and alternative pathway $\mathrm{C} 3$ convertases. DAF thereby blocks these two complement amplification convertase enzymes and provide protection from the subsequent complement cascade activation which involves the formation of $\mathrm{C} 5$ convertase and the membrane attack complex (C5b-9) that creates pores in membrane causing the cell lysis [15]. Our research has shown pharmacologic supplementation of DAF to be neuroprotective to blast effects by suppressing the systemic and local inflammatory response, improving blood-brain barrier 
integrity, reducing tau phosphorylation and decreasing cytotoxic edema [16]. DAF administration following hemorrhage and/or $\mathrm{I} / \mathrm{R}$ also has been shown to limit lung and intestine tissue damage, decrease complement activation and tissue deposition, decrease IL-6 production and improve survival chances [17-20]. We therefore hypothesized that DAF administration would ameliorate pulmonary and intestinal damage commonly produced by blast injury. In order to test this hypothesis, we administered rhDAF to rats that were subjected to BOP and assessed the lung and intestinal tissue injury benefits. We demonstrate in this study that DAF administration 30 minutes post-blast exposure decreases complement activation and mitigates tissue damage. Early administration of DAF to a victim of blast exposure may ameliorate intestinal and pulmonary injury and improve recovery.

\section{Materials and Methods}

\section{Animals}

Adult pathogen-free male Sprague-Dawley rats weighing 250 to $300 \mathrm{~g}$ (Charles River, Wilmington, MA) were used in this study. Experiments were conducted in compliance with the Animal Welfare Act at an AAALAS accredited institution and in accordance with the principles of the guide for the care and use of Laboratory Animals. The study was approved by the Joint WRAIR-NMRC Institutional Animal Care and Use Committee.

\section{Reagents}

Recombinant human DAF (rhDAF) and biotinylated anti-human DAF was obtained from R\&D systems (Minneapolis, MN). Rabbit polyclonal anti-caspase-1, anti-NE, and mouse anti-C5/C5a antibodies were obtained from Abcam Inc. (Cambridge, MA). Conjugated secondary antibodies (Streptavidin-Alexa Fluor 488, goat anti-mouse Alexa Fluor 488, goat anti-rabbit 594, and goat anti-chicken 594), and ProLong Gold anti-fade reagent were from Invitrogen (Carlsbad, CA).

\section{Experimental design and administration of DAF}

Rats were exposed to BOP as described previously [16]. Briefly, the rats were anesthetized with intra-peritoneal injection of ketamine/xylazine $(60 / 5 \mathrm{mg} / \mathrm{kg})$ and placed into the end of the expansion chamber of a compressed air-driven shock tube $(2.5 \mathrm{ft}$. diameter compression chamber connected to a $15 \mathrm{ft}$. expansion chamber) and immobilized to prevent movement from blast impact and subsequent secondary or tertiary blast injuries. Animals were subjected to a single blast exposure with mean peak overpressure of $120 \pm 7 \mathrm{kPa}$ with their right side ipsilateral to the direction of the BOP. Animals were randomly assigned to one of three experimental groups: 1) Control, animals underwent anesthesia, suspension, and time delays but no BOP exposure ( $\mathrm{n}=8$ per each time point of $3 \mathrm{~h}, 24 \mathrm{~h}$ and $48 \mathrm{~h}$ recovery); 2) BOP, animals were subjected to BOP followed by a bolus injection ( $0.5 \mathrm{ml}$ of saline) via tail vein $30 \mathrm{~min}$ post-BOP and recovered per specified time (BOP- $3 \mathrm{~h}, \mathrm{n}=8$; BOP- $24 \mathrm{~h}, \mathrm{n}=8$; BOP- $48 \mathrm{~h}$, $\mathrm{n}=5$ ); 3) DAF, animals were exposed to BOP followed by a bolus injection of $\mathrm{rhDAF}(50 \mu \mathrm{g} / \mathrm{kg}$ body weight in $0.5 \mathrm{~mL}$ saline) via tail vein 30 min post-BOP and recovered per specified time (DAF- $3 \mathrm{~h}, \mathrm{n}=6$; DAF-24h, $n=8$; DAF-48h, $n=5$ ). The animals in each group were euthanized at specified time points and lung and intestinal tissue samples from each animal were collected and sections of each were fixed with $10 \%$ formalin or $4 \%$ paraformaldehyde for histological and immunohistochemical analysis, respectively.

\section{Histological evaluation}

Ten percent formalin-fixed tissues were embedded in paraffin. Lung and intestine sections were then cut, and stained with Hematoxylin-Eosin (H\&E). Five random histologic images were recorded at $\times 400$ magnifications under an Olympus AX80 light microscope (Olympus, Center Valley, PA) and graded by a pathologist blinded to the treatment group. The two tissue-types were assessed as described previously [16] by five distinct morphological parameters: pathological and morphological changes (shrinkage of the cell body, pyknosis of the nucleus, disappearance of the nucleolus, loss of Nissl substance and eosinophilia of the cytoplasm, and cytotoxic edema). The changes were graded according to their extent (score 0, 1, 2, 3 and 4 for an extent of $0 \%,<25 \%, 25-50 \%, 50-75 \%$, and $75-100 \%$, respectively) and the severity of the injury (score $0=$ normal histology, score 1 =slight, $2=$ mild, $3=$ moderate and $4=$ severe alterations). The injury score represents the sum of the extent and the severity of injury and averaged per total number of animals in each group.

\section{Immunohistochemical staining}

After $4 \%$ paraformaldhyde fixation, lung tissue sections were transferred to $20 \%$ sucrose $(\mathrm{w} / \mathrm{v})$ in PBS overnight at $4^{\circ} \mathrm{C}$, followed by freezing in Tissue-Tex OCT mounting medium (Sakura, Netherlands). Frozen tissue sections were cut at $5 \mu \mathrm{m}$ thickness with a cryostat and mounted onto glass slides. Tissues were fixed in $4 \%$ paraformaldehyde for $20 \mathrm{~min}$ and permeabilized with $0.2 \%$ Triton X-100 in PBS for $10 \mathrm{~min}$. Sections were blocked with $2 \%$ bovine serum albumin and incubated with primary antibodies overnight at $4^{\circ} \mathrm{C}$. After washing, sections were incubated with the appropriate secondary antibodies labeled with Alexa Fluor 488 or 594 for $1 \mathrm{~h}$ at room temperature. After washing, sections were mounted with ProLong Gold antifade solution containing 4, 6'-diamidino-2-phenylindole and visualized under a Radiance 2100 confocal laser scanning microscope (Bio-Rad, Hercules, CA) at $\times 200$ or $\times 400$ magnification. Negative controls were conducted by substituting the primary antibodies with corresponding immunoglobulin isotypes. Captured digital images were processed by Image J software (NIH, Bethesda, MD).

\section{Immunofluorescent quantification}

This procedure is based on a modified method as described previously [21]. Briefly, four to six images from each animal section were calibrated and adjusted using Adobe Photoshop software until only the fluorescent deposits were visible with no visible tissue background. Image J software was then utilized and the image was changed to black and white pixels with black representing deposits of the target proteins and white representing nonstained areas of the image. Using the image Adjust Threshold command, the image was then changed to red and white (fluorescent deposits were in red). Image analysis resulted in the red total area in pixels squared. Densitometric values for total area for all animal sections in each group which were then used to determine the average area of fluorescent deposit.

\section{Statistical analysis}

Data are expressed as mean \pm Standard Error of the Mean (SEM). One-way Analysis of Variance (ANOVA) followed by Bonferroni or unpaired t-test was performed using GraphPad Prism ${ }^{\circledast}$ (5.0, GraphPad Software, San Diego, CA). P value $<0.05$ was considered as significant. 


\section{Results}

\section{Recombinant human DAF deposits in pulmonary tissue}

Deposition of rhDAF in the lung was determined by immunofluorescence staining using anti-human DAF antibody. While in the control and BOP groups no rhDAF deposition was noted, in the lungs of BOP+DAF-treated animals, the rhDAF deposition as evidenced by the red signal was observed (Figure 1). These rhDAF depositions appeared to be primarily associated with alveolar endothelial cells.
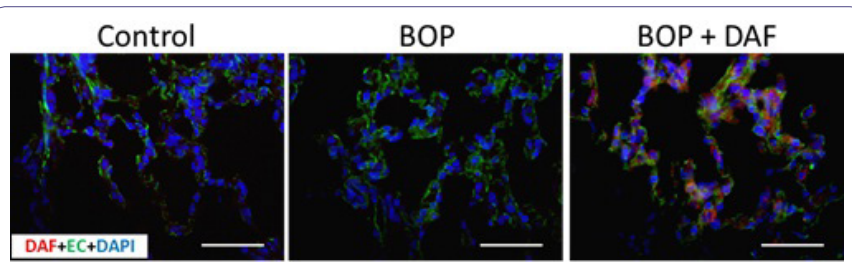

Figure 1: Deposition of rhDAF in the rat lung. Representative photomicrographs of the frozen sections from rat lungs immunostained by anti-human DAF (red), anti-Endothelial Cell (EC, green) antibodies, and DAPI (4', 6-Diamidino-2-phenylindole dihydrochloride). Original magnification of $x$ 200. Scale bar, $200 \mu \mathrm{m}$. $\mathrm{n}=8$ for both control and experimental groups.

\section{Administration of rhDAF mitigates pulmonary tissue injury in a murine model of $\mathrm{BOP}$}

Histological analyses of the lung after a recovery period of 3, 24 and $48 \mathrm{~h}$ following blast exposure revealed microscopic changes in the alveolar epithelial cells (Figure 2). BOP exposure resulted in tissue injuries of bilateral capillary damage (Figure 2, red arrow), infiltration of inflammatory cells, lung edema and bronchi morphological change characterized by cell body shrinkage and nuclear pyknosis. These injury changes were significantly attenuated by an early bolus administration of rhDAF, $30 \mathrm{~min}$ post-BOP. The beneficial effects of DAF administration were seen throughout the recovery periods of 3, 24 and $48 \mathrm{~h}$ post-blast exposure. The beneficial effects of rhDAF were so substantial at $24 \mathrm{~h}$, that the results of histological damage and injury score analysis of DAF administrated group were very similar to the control group.

\section{Administration of rhDAF mitigates intestinal tissue injury in a murine model of BOP}

Histological analyses of the epithelial cell mucosa after a recovery period of 3, 24 and $48 \mathrm{~h}$ following BOP revealed microscopic injuries in the enterocytes located on top of villi and revealed significant changes in morphology in comparison to controls (Figure 3 ). These changes were characterized by notable structural loss of microvilli numbers and size alteration with morphologic features consisting of shrinkage of cell body, pyknosis of nucleus, disappearance of nucleolus, and loss of Nissl substances. Mucosa showed apparent damage to lamina propria (submucosa) with villi edema and inflammatory cell infiltration. These injury changes were significantly attenuated by an early bolus administration of rhDAF. The beneficial effects of DAF administration were seen throughout the recovery periods of $3 \mathrm{~h}, 24 \mathrm{~h}$ and $48 \mathrm{~h}$ after the blast exposure. Similar to the lung data above, the rhDAF beneficial effects were so evident in the intestinal tissue as well, that the injury scores of the DAF administrated groups at both $24 \mathrm{~h}$ and $48 \mathrm{~h}$, showed no difference compared to their respective controls.

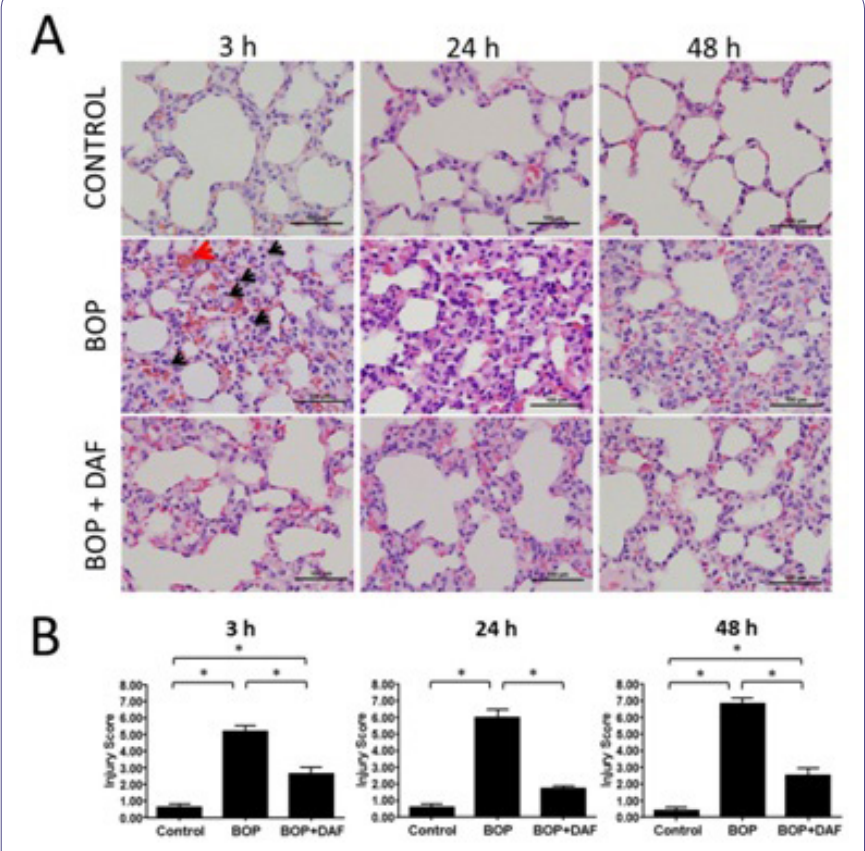

Figure 2: Early treatment of rhDAF attenuates lung tissue injury in a murine model of BOP. A) All were representative photomicrographs of lung paraffin sections with H\&E of BOP-3h, BOP-24h and BOP-48h control, untreated and treated with $\mathrm{rhDAF}(50 \mu \mathrm{g} / \mathrm{kg}) 30 \mathrm{~min}$ post blast exposure. Original magnification of $x 400$. Scale bar, $100 \mu \mathrm{m}$. B) Mean lung injury scores were graphed. $n=8$ for all control, BOP-3h and BOP-24h groups and $n=5$ for BOP-48h group. $n=6,8$ and 5 for BOP+DAF-3h, BOP+DAF-24h and $\mathrm{BOP}+\mathrm{DAF}-48 \mathrm{~h}$ respectively. Injury scores calculated using the criteria as described in the Methods. Graphical data expressed as mean \pm SEM, and compared using one-way ANOVA followed by Bonferroni test.

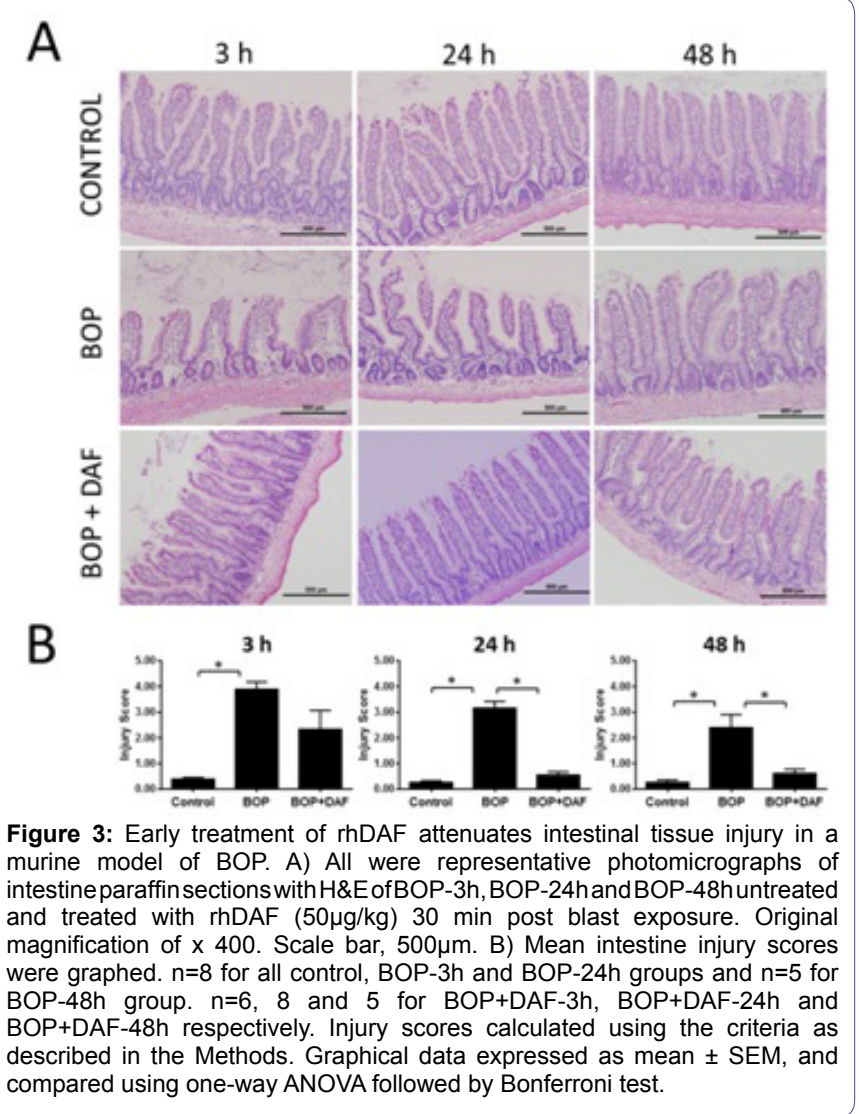


Citation: Campbell JE, Oh T, Hurtgen BJ, Niemeyer DM, Sheppard FR, et al. (2015) Blast Overpressure Induced Pulmonary and Intestinal Damage is Ameliorated by Post-injury Decay Accelerating Factor Injection. J Clin Immunol Immunother 2: 007.

\section{rhDAF attenuates complement expression and deposition in} lung tissue promoted by BOP injury

The complement component C5 was deposited at the epithelial layer of the alveoli as early as $3 \mathrm{~h}$ and lasted at least up to $24 \mathrm{~h}$ after BOP as compared to the control (Figure 4). The mean immunofluorescence intensities of the BOP group were significantly higher at both 3 and $24 \mathrm{~h}$ compared to the respective control groups. More importantly, the administration of rhDAF, significantly decreased this deposition of $\mathrm{C} 5$ in the lung tissues at both $3 \mathrm{~h}$ and $24 \mathrm{~h}$ and to the levels that are comparable to the respective controls.

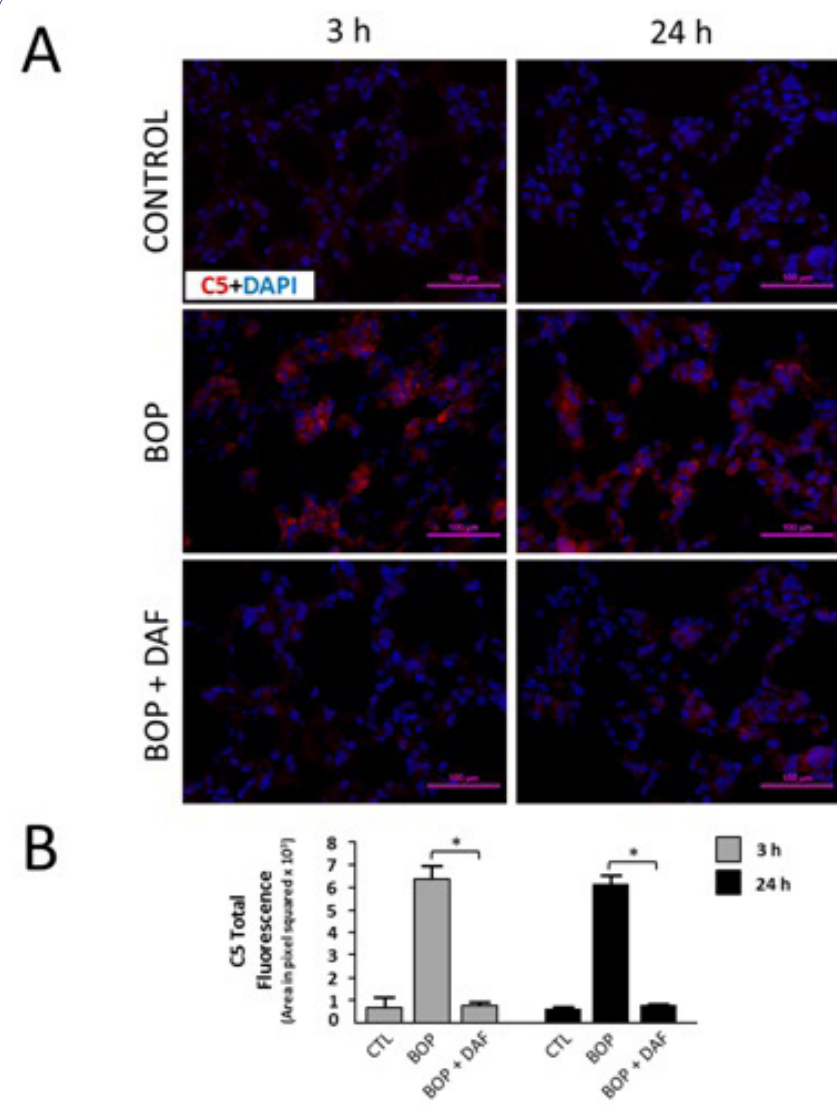

Figure 4: rhDAF decreases $\mathrm{C} 5 / \mathrm{C} 5 \mathrm{a}$ deposition in lung tissue from BOP exposed rats. A) Representative photomicrographs of the lung frozen sections were stained with anti-C5/C5a antibodies and DAPI. Original magnification of $x 400$. Scale bar, $100 \mu \mathrm{m}$. B) Mean fluorescence intensities were graphed. $n=8$ for all control, BOP-3h and BOP-24h groups. $n=6$ and 8 for BOP+DAF-3h and BOP+DAF-24h respectively. Total immunofluorescences were calculated using the criteria as described in the Methods. Graphical data expressed as mean \pm SEM, and compared using one-way ANOVA followed by Bonferroni test.

\section{rhDAF reduces $\mathrm{NE}$ deposition in lung tissue in a murine model of BOP}

Significant increases in the deposition of NE within the lungs after BOP exposure were observed at both $3 \mathrm{~h}$ and $24 \mathrm{~h}$ time points studied (Figure 5). The administration of rhDAF dramatically prevented the deposition of NE in the respiratory organ after BOP exposure and the immunofluorescence intensities at both $3 \mathrm{~h}$ and $24 \mathrm{~h}$ after rhDAF administration were very similar to that of the matched controls.

rhDAF prevents increases in caspase-1 levels in the lung following BOP exposure

In the lungs, the levels of caspase- 1 at the $24 \mathrm{~h}$ time point was significantly elevated over sham-operated control animals
A
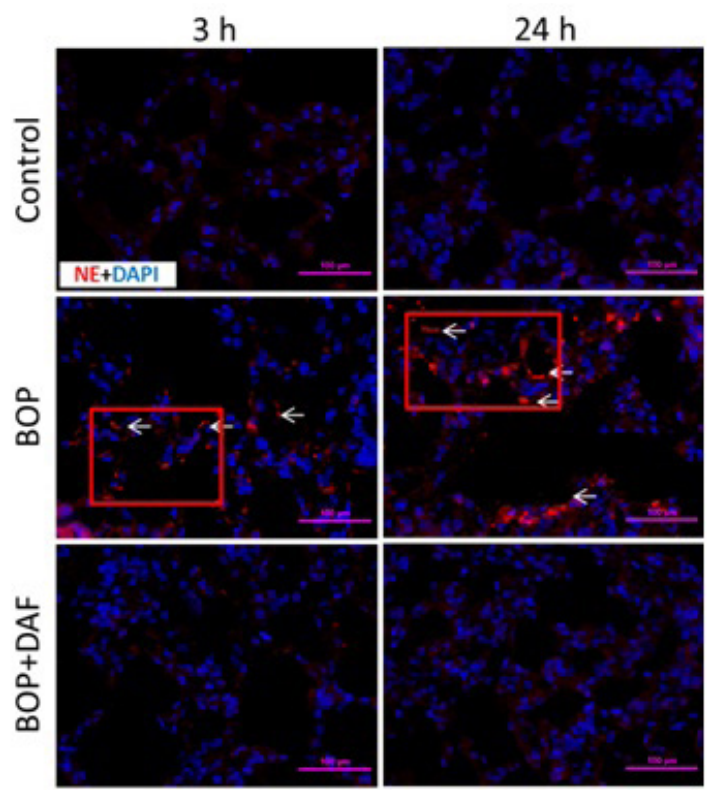

B

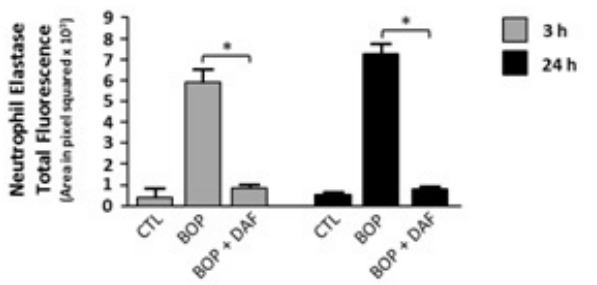

Figure 5: rhDAF decreases NE deposition in lung tissue from BOP exposed rats. A) Representative photomicrographs of the lung frozen sections were stained with anti-NE antibodies and DAPI. Original magnification of $x 400$ Scale bar, $100 \mu \mathrm{m}$. Red boxes indicate region of interest for measurement whereas white arrows represent clusters of activity rather than ubiquitous activation. B) Mean fluorescence intensities were graphed. $n=8$ for all control, BOP-3h and BOP-24h groups. $n=6$ and 8 for BOP+DAF-3h and BOP+DAF-24h respectively. Total immunofluorescences were calculated using the criteria as described in the Methods. Bar graph values were expressed as mean \pm SEM and compared using one-way ANOVA followed by a Bonferroni test.

(Figure 6). DAF administration following BOP prevented this significant increase in caspase-1 levels. No significant differences were evident in the $3 \mathrm{~h}$ or $48 \mathrm{~h}$ time point's \pm DAF.

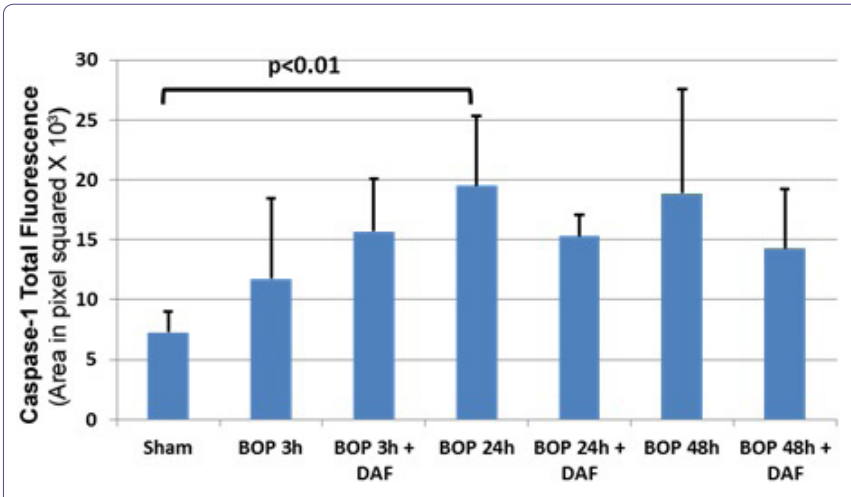

Figure 6: Caspase-1 was increased in the respiratory organ from BOP exposed rats. Mean fluorescence intensities were graphed. $n=8$ for sham control, BOP-3h and BOP-24h groups and $n=5$ for BOP-48h group. $n=6,8$ and 5 for BOP+DAF-3h, BOP+DAF-24h and BOP+DAF-48h respectively. Total immunofluorescences were calculated using the criteria as described in the Methods. Graphs expressed as mean \pm SEM, and compared using one-way ANOVA followed by Bonferroni test. 


\section{Discussion}

Blast-injury is a real and present danger for the civilian population and a greater danger to those exposed to military conflict [22]. Clinicians have very little experience in treating blast overpressure-injured patients and the best way forward for immediate and continued treatment is unknown [12]. We demonstrated in a previous manuscript that recombinant human Decay Accelerating Factor (rhDAF) administration protected a BOP animal model against Blast-Induced Neurotrauma (BINT) by suppression of both systemic and local inflammatory responses $[16,20,23]$. As a result, modulation of complement activation after simulated blast injury was hypothesized as a novel and significant therapeutic tool aimed at injury prevention via administration of rhDAF.

In this manuscript, we continued to evaluate the beneficial effects of complement inhibition using DAF for prevention of BOP-induced inflammatory damage to the lung and intestine, two highly susceptible organs to blast injury. There were several major findings. 1) Our model of moderate blast exposure $(120 \mathrm{kPa})$ induces histopathological, structural, and morphological disruptions and damage that was significantly elevated at 3,24 , and $48 \mathrm{~h}$ post-BOP exposure per injury score assessment. 2) Intravenous rhDAF administration $30 \mathrm{~min}$ post-BOP exposure significantly mitigated BOP induced immediate ( 3 hours post-BOP) and delayed injury (24 hours and 48 hours post-BOP) (Figure 2). 3) Our data showed rhDAF deposition upon the surface of lung tissues (Figure 1) and suggest the beneficial mechanism of rhDAF treatment occurred via reduced $\mathrm{BOP}$ activation of $\mathrm{C} 5$ or competitive inhibition of $\mathrm{C} 5$ deposition (Figure 4), and reduction of neutrophil elastase as a marker of inflammation (Figure 5). The data presented support our hypothesis that DAF administration post-BOP ameliorates pulmonary and intestinal damage commonly produced by blast injury.

rhDAF administration promotes preservation of normal alveoli and villi structures following BOP injured lung and intestine. This finding, when considered in combination with our data of no significant differences in the mRNA expression of complement $\mathrm{C} 3 / \mathrm{C} 3 \mathrm{a}$ and $\mathrm{C} 5 / \mathrm{C} 5 \mathrm{a}$ as well as inflammatory markers IL- $1 \beta$ and TNF $\alpha$ (data not shown) between BOP and BOP+DAF groups, supports the mechanism of DAF tissue preservation as being in agreement with its previously characterized function. DAF does not interfere with lower orders of complement activation but rather prevents enzyme complex assembly upon a cellular surface [15]. However, DAF may interrupt $\mathrm{C} 3 \mathrm{a}-\mathrm{C} 3 \mathrm{aR}$ and/or Ca-C5aR signal pathways for inhibition of cell death [24], which is supported by our findings that a trend of less caspase-1 levels with DAF administration ( $p>0.05$, figure 6). Additionally, these signaling pathways are involved in neutrophil recruitment and activation [25], which is supported by our decrease in detection of NE in the lungs following rhDAF administration (Figure 5).

Pulmonary and intestinal tissue preservation in the trauma scenario represents tremendous leverage in survival outcomes as increase in mortality strongly correlates with Multi-Organ Dysfunction (MOD) and Failure (MOF) [26,27]. Although BOP has not been studied or shown to precipitate into MOD/F, BOP rarely occurs as a singular insult. Accompanying secondary and tertiary blast injuries contribute to the propensity for major trauma and blood loss that may lead to $\mathrm{MOD} / \mathrm{F}$. When it comes to $\mathrm{MOD} / \mathrm{F}$, prevention is the best medicine [28] which is seemingly the case with DAF administration following BOP exposure. Neutrophil Elastase (NE) data in this study cannot be clinically correlated yet strong evidence supports NE in the pathophysiology of acute lung injury and in the development of acute respiratory distress syndrome [29]. DAF significantly diminished NE concentration following BOP (Figure 5) and may therefore represent a partial mechanism for the decrease in severity of lung injury. The precise mechanism is unknown but may be related to suppression of neutrophil activation and/or inhibition of neutrophil recruitment. Injury-preventing results of DAF administration following blast are consistent with our findings of DAF therapy-induced decreases in tissue injury following hemorrhagic shock $[17,18]$ and ischemia/reperfusion injury [19].

\section{Conclusion}

DAF protects lung and intestine from BOP-induced injury by direct suppression of $\mathrm{C} 5 / \mathrm{C} 5 \mathrm{a}$ deposition and therefore assembly of complement enzymatic complexes. The observed improvements in tissues investigated in the groups that received DAF administration suggest similar improvements may also be gained with DAF therapy following blast exposure in humans. DAF may represent an attractive option for use by first-responders or emergency medical technicians for improved preservation of pulmonary and intestinal tissues and allow for patient arrival at a primary point of care in a lesser critical condition.

\section{Disclosures}

The views expressed in this article are those of the author and do not necessarily reflect the official policy or position of the Department of the Air Force, Department of the Navy, US Army, Department of Defense, or the US Government.

The study protocol was reviewed and approved by the Walter Reed Army Institute of Research/Naval Medical Research Center Institutional Animal Care and Use Committee in compliance with all applicable Federal regulations governing the protection of animals in research.

The authors are military members or employee of the US Government. This work was prepared as part of their official duties. Title 17 U.S.C. $\$ 105$ provides that "Copyright protection under this title is not available for any work of the United States Government." Title 17 U.S.C. $\$ 101$ defines a US Government work as a work prepared by a military service member or employee of the US Government as part of that person's official duties. The authors declare no competing interests or conflicts of interest.

\section{Acknowledgements}

Authorship: JJD designed the study. JEC, TO, and BJH analyzed and interpreted data. JJD, DN, and FRS supplied critical evaluation. JEC, TO, and BJH drafted the paper. This work was supported by a grant from the US Army Medical Research and Materiel Command, Fort Detrick, MD. The authors are thankful to Lingamanaidu $\mathrm{V}$ Ravichandran, PhD for critical review and, for the help in the revision and editorial assistance with the manuscript.

\section{References}

1. Hooker DR (1924) Physiologic effects of air concussion. American Journal of Physiology 67: 219-274.

2. Zuckermann S (1940) Experimental study of blast injury to lungs. Lancet 236 : 219-224.

3. O'Reilly JN, Gloyne SR (1941) Blast Injury of the Lungs. The Lancet 238: 423-428. 
4. Fung YC, Yen RT, Tao ZL, Liu SQ (1988) A hypothesis on the mechanism of trauma of lung tissue subjected to impact load. J Biomech Eng 110: 50-56.

5. Irwin RJ, Lerner MR, Bealer JF, Lightfoot SA, Brackett DJ, et al. (1998) Global primary blast injury: a rat model. J Okla State Med Assoc 91: 387-392.

6. Wani I, Parray FQ, Sheikh T, Wani RA, Amin A, et al. (2009) Spectrum of abdominal organ injury in a primary blast type. World J Emerg Surg 4: 46.

7. Stuhmiller JH (2010) Blast injury: Translating research into operational medicine. In: FriedI K, Santee WR (eds.). Military quantitative physiology: problems and concepts in military operational medicine. Borden Institute Monograph, USA.

8. Hepper AE, Pope DJ, Bishop M, Kirkman E, Sedman A, et al. (2014) Modelling the blast environment and relating this to clinical injury: experience from the 7/7 inquest. J R Army Med Corps 160: 171-174.

9. Radford P, Patel HD, Hamilton N, Collins M, Dryden S (2011) Tympanic membrane rupture in the survivors of the July 7,2005 , London bombings. Otolaryngol Head Neck Surg 145: 806-812.

10. de Ceballos JP, Turegano-Fuentes F, Perez-Diaz D, Sanz-Sanchez M, Martin-Llorente C, et al. (2005) 11 March 2004: The terrorist bomb explosions in Madrid, Spain-an analysis of the logistics, injuries sustained and clinical management of casualties treated at the closest hospital. Critical Care 9: 104-111.

11. Pan PP (2010) Russia braces for terrorism's return as 38 die in subway bombings. The Washington Post.

12. CDC (2003) Explosions and blast injuries: A primer for clinicians. US Department of Health \& Human Services, GA, USA.

13. Dalle Lucca JJ, Li Y, Simovic M, Pusateri AE, Falabella M, et al. (2012) Effects of $\mathrm{C} 1$ inhibitor on tissue damage in a porcine model of controlled hemorrhage. Shock 38: 82-91.

14. Ioannou A, Lieberman LA, Dalle Lucca JJ, Tsokos GC (2013) Complement depletion protects lupus-prone mice from ischemia-reperfusion-initiated organ injury. American Journal of Physiology - Gastrointestinal \& Liver Physiology 304: 283-292.

15. Fujita T, Inoue T, Ogawa K, lida K, Tamura N (1987) The mechanism of action of Decay-Accelerating Factor (DAF). DAF inhibits the assembly of C3 convertases by dissociating C2a and Bb. J Exp Med 166: 1221-1228.

16. Li Y, Chavko M, Slack JL, Liu B, McCarron RM, et al. (2013) Protective effects of decay-accelerating factor on blast-induced neurotrauma in rats. Acta Neuropathol Commun 1: 52.
17. Dalle Lucca JJ, Li Y, Simovic MO, Slack JL, Cap A, et al. (2013) Decay-accelerating factor limits hemorrhage-instigated tissue injury and improves resuscitation clinical parameters. J Surg Res 179: 153-167.

18. Dalle Lucca JJ, Simovic M, Li Y, Moratz C, Falabella M, et al. (2011) Decay-accelerating factor mitigates controlled hemorrhage-instigated intestinal and lung tissue damage and hyperkalemia in swine. J Trauma 71: 151-160.

19. Lu X, Li Y, Simovic MO, Peckham R, Wang Y, et al. (2011) Decay-accelerating factor attenuates $C$-reactive protein-potentiated tissue injury after mesenteric ischemia/reperfusion. J Surg Res 167: 103-115.

20. Wang Y, Li Y, Dalle Lucca SL, Simovic M, Tsokos GC, et al. (2010) Decay accelerating factor (CD55) protects neuronal cells from chemical hypoxia-induced injury. J Neuroinflammation 7: 24.

21. Weeks C, Moratz C, Zacharia A, Stracener C, Egan R, et al. (2007) Decay-accelerating factor attenuates remote ischemia-reperfusion-initiated organ damage. Clin Immunol 124: 311-327.

22. Mackenzie IM, Tunnicliffe B (2011) Blast injuries to the lung: epidemiology and management. Philos Trans R Soc Lond B Biol Sci 366: 295-299.

23. Dalle Lucca JJ, Chavko M, Dubick MA, Adeeb S, Falabella MJ, et al. (2012) Blast-Induced moderate Neurotrauma (BINT) elicits early complement activation and Tumor Necrosis Factor alpha (TNFa) release in a rat brain. $\mathrm{J}$ Neurol Sci 318: 146-154.

24. Salter MW, Kalia LV (2004) Src kinases: a hub for NMDA receptor regulation. Nat Rev Neurosci 5: 317-328.

25. Guo RF, Ward PA (2005) Role of C5a in inflammatory responses. Annu Rev Immunol 23: 821-852.

26. Fry DE (1988) Multiple system organ failure. Surg Clin North Am 68: 107-122.

27. Khwannimit B (2008) Serial evaluation of the MODS, SOFA and LOD scores to predict ICU mortality in mixed critically ill patients. J Med Assoc Thai 91: $1336-1342$

28. Baue AE (1999) Sepsis, Multi-Organ Dysfunction Syndrome (MODS) and Multiple Organ Failure (MOF). Prevention is better than treatment. Minerva Anestesiol 65: 477-480.

29. Kawabata K, Hagio T, Matsuoka S (2002) The role of neutrophil elastase in acute lung injury. Eur J Pharmacol 451: 1-10. 